\title{
Beam-width-dependent filtering properties of strong volume holographic gratings
}

\author{
Hung-Te Hsieh, Wenhai Liu, Frank Havermeyer, Christophe Moser, and Demetri Psaltis
}

\begin{abstract}
The finite dimension of the incident beam used to read out volume holographic gratings has interesting effects on their filtering properties. As the readout beam gets narrower, there is more deviation from the ideal response predicted for monochromatic plane waves. In this paper we experimentally explore beam-width-dependent phenomena such as wavelength selectivities, angular selectivities, and diffracted beam profiles. Volume gratings in both reflection and transmission geometries are investigated near $1550 \mathrm{~nm}$. Numerical simulations utilizing the technique of Fourier decomposition provide a satisfactory explanation and confirm that the spread of spatial harmonics is the main contributing factor. (C) 2006 Optical Society of America

OCIS codes: $\quad 050.7330,090.2890,090.7330$.
\end{abstract}

\section{Introduction}

A volume holographic grating (VHG) consists of a periodically modulated refractive index pattern imprinted within a bulk recording material, e.g., glasses, ${ }^{1}$ photorefractive crystals, ${ }^{2}$ and photosensitive polymers. ${ }^{3}$ Without loss of generality, such a grating can be represented as

$$
n(\mathbf{r})=n+\Delta n \cos \mathbf{K} \cdot \mathbf{r},
$$

where $\mathbf{K}$ is the grating vector, $n$ is the (average) refractive index of the medium, and $\Delta n$ stands for the index modulation depth as a result of the recording process.

Plane waves are the eigenmodes in an unperturbed, homogeneous medium and VHGs are capable of efficiently coupling plane waves with different wave vectors when energy and momentum are conserved. The maximum coupling strength is achieved between the incident and the diffracted waves when the Bragg condition

$$
\mathbf{K}=\mathbf{k}_{\mathbf{i}}-\mathbf{k}_{\mathbf{d}}
$$

H.-T. Hsieh (tete@caltech.edu) and D. Psaltis are with the Department of Electrical Engineering at the California Institute of Technology, Pasadena, California 91125. W. Liu, F. Havermeyer, and C. Moser are with Ondax Incorporated, 850 East Duarte Road, Monrovia, California 91016.

Received 4 April 2005; accepted 25 September 2005.

0003-6935/06/163774-07\$15.00/0

(C) 2006 Optical Society of America is satisfied, where $\mathbf{k}_{\mathbf{i}}$ and $\mathbf{k}_{\mathbf{d}}$ are the wave vectors of the incident and diffracted waves, respectively. The principle of optical filtering by a VHG is therefore based on the distinction between optical fields that satisfy the Bragg condition and those that do not. Operation with free space optics further renders it an extremely versatile tool for optical information processing.

The coupling effects between plane waves mediated by VHGs have been treated rigorously with various theoretical approaches, e.g., coupled-mode analysis ${ }^{4,5}$ and the matrix method. ${ }^{6}$ We will use these well-established results as given for our numerical simulations.

Unlike thin gratings, VHGs are very sensitive to phase mismatch because of less ambiguity allowed in the momentum space. Thanks to its excellent selectivity, VHGs have become an ideal candidate for various promising technological applications such as neural networks, ${ }^{7}$ optical correlators, ${ }^{8}$ and holographic data storage. ${ }^{9,10}$ VHGs also prove useful in telecommunications; essentially a one-dimensional photonic crystal when $\Delta n$ is appreciable $(\Delta n \geq$ $10^{-4}$ ), holographic gratings in the reflection geometry can serve as superior filters ${ }^{11,12}$ for wavelength division multiplexing (WDM) thanks to their low cross talk and readily engineered bandwidths.

The versatility of VHGs provides the motivation behind our work presented here. In this paper we show the experimentally measured beam-width dependence of the wavelength selectivity, angular selectivity, and diffracted beam profiles of VHGs in 
both the reflection and the transmission geometries for $s$-polarized fields. With the help of Fourier analysis, we can satisfactorily explain the interesting discrepancies between the experimental data and the ideal results predicted for plane waves.

\section{Theoretical Consideration}

In Fig. 1, we consider how a VHG affects a monochromatic incident optical field whose complex amplitude is $E_{i}(x, y, z)$. $\mathrm{VHG}_{\mathrm{R}-\mathrm{T}}$ represents a volume holographic grating in the reflection-transmission geometry. The grating length measures $L$ along the $z$ axis and its $x-y$ cross section (much larger than the beam area) can be regarded as infinite for all practical cases. With the knowledge of $f(x, y)=E_{i}(x, y, z$ $=0)$, the complex amplitude of the incident beam $E_{i}(x, y, z)$ can be uniquely determined by the diffraction integral in the case of propagation in a homogeneous medium:

$$
\begin{aligned}
E_{i}(x, y, z)= & \iint F\left(k_{i x}, k_{i y}\right) \exp \left[-j\left(k_{i x} x+k_{i y} y\right.\right. \\
& \left.\left.+k_{i z} z\right)\right] \frac{\mathrm{d} k_{i x}}{2 \pi} \frac{\mathrm{d} k_{i y}}{2 \pi},
\end{aligned}
$$

where $k_{i}=\left|\mathbf{k}_{\mathbf{i}}\right|=\left(k_{i x}{ }^{2}+k_{i y}{ }^{2}+k_{i z}{ }^{2}\right)^{1 / 2}$ is the wavenumber of the monochromatic radiation and $F\left(k_{i x}, k_{i y}\right)$ is the 2D Fourier transform of $f(x, y)$. Each $F\left(k_{i x}, k_{i y}\right)$ represents a constituent plane-wave (or angular) component of $E_{i}(x, y, z)$. Basic Fourier-transform relationships dictate that the narrower the incident beam is, the more spread out its angular components will be. The wavefront nonuniformity of $E_{i}(x, y, z)$ can be thought of as a natural manifestation of the linear combination of all its plane-wave elements $F\left(k_{i x}, k_{i y}\right) e^{-j \mathbf{k}_{\mathbf{i}} \cdot \mathbf{r}}$. For an obliquely incident optical field (tilted by $\theta$ ), such as $E_{i}{ }^{\prime}(x, y, z)$ in Fig. 1, a simple change of variable can be used to simplify the math-

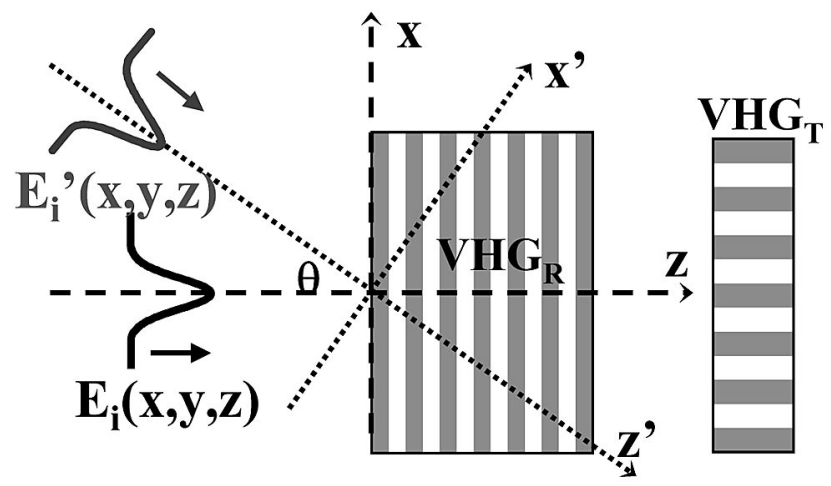

Fig. 1. Theoretical configuration. The volume holographic grating has a transfer function $H\left(\mathbf{k}_{\mathbf{i}} ; \mathbf{k}_{\mathrm{d}}\right) . \mathrm{VHG}_{\mathrm{R}-\mathrm{T}}$ is a volume holographic grating in the reflection-transmission geometry.

$H\left(\mathbf{k}_{\mathbf{i}} ; \mathbf{k}_{\mathrm{d}}\right)$ provides the opportunity for some of the incident plane-wave components $F\left(k_{i x}, k_{i y}\right) e^{-j \mathbf{k}_{\mathbf{i}} \cdot \mathbf{r}}$ in the incident field to be diffracted and gives rise to the (diffracted) plane-wave components:

$$
F\left(k_{i x}, k_{i y}\right) H\left(\mathbf{k}_{\mathbf{i}} ; \mathbf{k}_{\mathbf{d}}\right) e^{-j \mathbf{k}_{\mathbf{d}} \cdot \mathbf{r}} .
$$

Since this is a linear system, the diffracted beam will be the integral sum of all diffracted plane waves

$$
E_{d}(x, y, z)=\iint F\left(k_{i x}, k_{i y}\right) H\left(\mathbf{k}_{\mathbf{i}} ; \mathbf{k}_{\mathbf{d}}\right) e^{-j \mathbf{k}_{\mathbf{d}} \cdot \mathbf{r}} \frac{\mathrm{d} k_{i x}}{2 \pi} \frac{\mathrm{d} k_{i y}}{2 \pi}
$$

Placing a detector (e.g., $D_{\text {diff }}$ in the experimental setup as depicted in Fig. 2) at the output plane $\sum_{o}$ to measure the diffracted field intensity, the diffraction efficiency $\eta$ can be calculated from the diffracted and transmitted field intensities:

The derivation of the appropriate transfer function

$$
\eta=\frac{\iint_{\Sigma_{o}}\left|E_{d}(x, y, z)\right|^{2} \mathrm{~d} \Sigma_{o}}{\iint\left|E_{i}\left(x^{\prime}, y, z^{\prime}=L\right)\right|^{2} \mathrm{~d} x^{\prime} \mathrm{d} y+\iint_{\Sigma_{o}}\left|E_{d}(x, y, z)\right|^{2} \mathrm{~d} \Sigma_{o}} .
$$

ematical expressions

$$
x^{\prime}=x \cos \theta+z \sin \theta, \quad z^{\prime}=-x \sin \theta+z \cos \theta .
$$

The angle of incidence $\theta$ is the angle between the beam propagation direction and the normal of the input face $z=0$. Unless noted otherwise, $\theta$ is measured inside the VHG.

The presence of the VHG with frequency response
$H\left(\mathbf{k}_{\mathbf{i}} ; \mathbf{k}_{\mathbf{d}}\right)$ of a VHG has been treated by an abundance of literature ${ }^{4-6}$ and will be used as given in our simulations.

\section{Numerical Simulations and Experimental Results}

Here we present the experimental results and numerical simulations of VHGs in the reflection and transmission geometries separately. In all cases the electric fields are perpendicular to the plane of inci- 


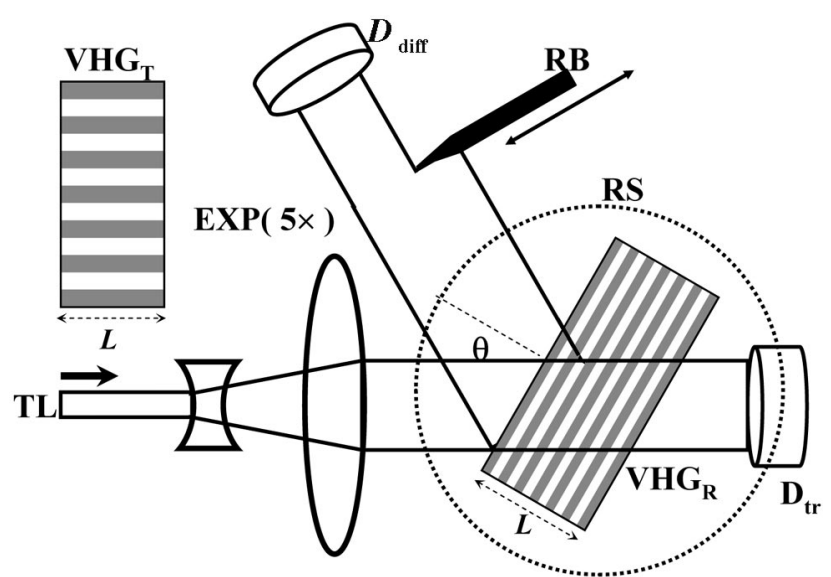

Fig. 2. Experimental setup: TL, tunable laser source (from 1520 to $1600 \mathrm{~nm}$ ); $\operatorname{EXP}(5 \times)$; beam expander; $\mathrm{VHG}_{\mathrm{R}-\mathrm{T}}$, volume holographic grating in the reflection-transmission geometry; $D_{\text {diff }}$, detector for the transmitted-diffracted beam; RS, rotational stage: $\mathrm{RB}$, razor blade controlled by a translation stage for measurement of the diffracted beam profile.

dence ( $s$ polarized). For each geometry we first describe the experimental setup and then compare the measurements and theoretical predictions for two different beam widths. All VHGs used were provided by Ondax Incorporated and recorded on photosensitive glass plates. The absorption of the glass is negligible.

\section{A. Reflection Geometry}

In telecommunications, WDM filters are needed to select and/or manipulate a desired wavelength from a bank of available channels. In reconfigurable communication systems, tunable optical filters play an increasingly important part. Examples include tunable arrayed waveguide gratings (AWGs), ${ }^{13}$ wavelength tuning based on varying temperature, ${ }^{14}$ and the application of stress. ${ }^{15}$ Tunable filters have also been realized in reflection-geometry VHGs by means of angular tuning. ${ }^{16}$ To appreciate and efficiently utilize this filtering configuration, it is important to know how oblique incidence impacts the filtering properties.

\section{Experimental Setup}

The experimental setup is schematically shown in Fig. 2; a reflection-geometry $\mathrm{VGH}, \mathrm{VGH}_{R}$, whose grating period $\Lambda$ is approximately $532 \mathrm{~nm}$ (the Bragg wavelength at normal incidence is approximately $1581 \mathrm{~nm}$ ) is mounted on a rotational stage for precise angular control. The light from a tunable laser source (tuning range 1520-1600 nm) is channeled through a fiber collimator (Newport Model f-col-9-15) and used to conduct measurements. The interaction length at normal incidence $L$ is $14 \mathrm{~mm}$. The output laser beam profile from the collimator is Gaussian with a diameter $W$ of $0.5 \mathrm{~mm}$, which is the spatial width across its intensity profile where it drops to $1 / e^{2}$ of the peak value. A beam expander $(5 \times)$ consisting of two cylindrical lenses (a negative lens with a focal length of $-15 \mathrm{~mm}$ and a positive lens with a focal length of $75 \mathrm{~mm}$ ) can be moved in to widen the incident beam. The angular spread of the (un-)expanded beam is calculated to be $\approx\left(0.15^{\circ}\right) 0.03^{\circ}$ inside the glass, corresponding to $\approx\left(0.225^{\circ}\right) 0.045^{\circ}$ in the air.

For each incident angle $\theta$ and wavelength $\lambda$ of interest, the power of both the transmitted and the diffracted beams are monitored, from which the diffraction efficiency can be calculated. A razor blade that is motion controlled by a translation stage can be moved across the diffracted beam to measure its intensity profile. The distance between the razor blade and the output face is approximately $50 \mathrm{~mm}$.

\section{Wavelength Selectivity}

To measure wavelength selectivity curves, incident angle $\theta$ is first set to one of a series of predetermined values, from $0^{\circ}$ to $13.3^{\circ}$, and then a wavelength scan is carried out. Figure 3(a) shows the results obtained with the narrow, unexpanded beam $B_{N} \quad(W$ $=0.5 \mathrm{~mm})$; the transmittance $(=1-\eta)$ curves are plotted in decibels. Each valleylike feature corresponds to strong coupling of the incident beam to the

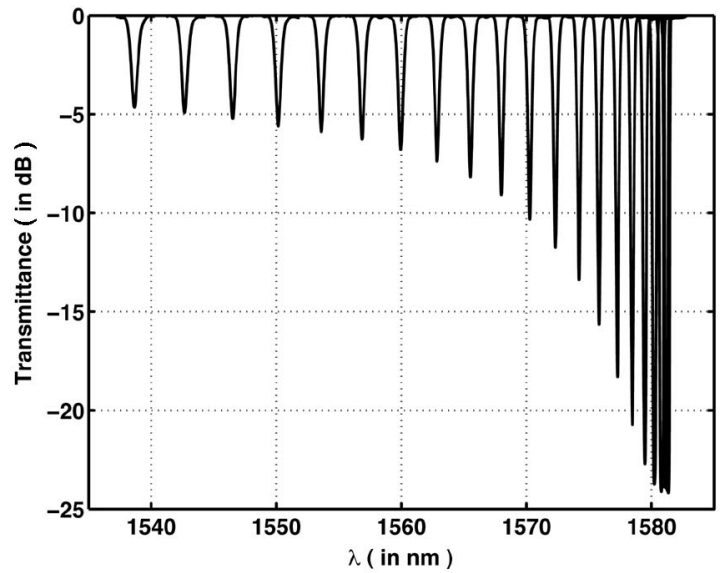

(a)

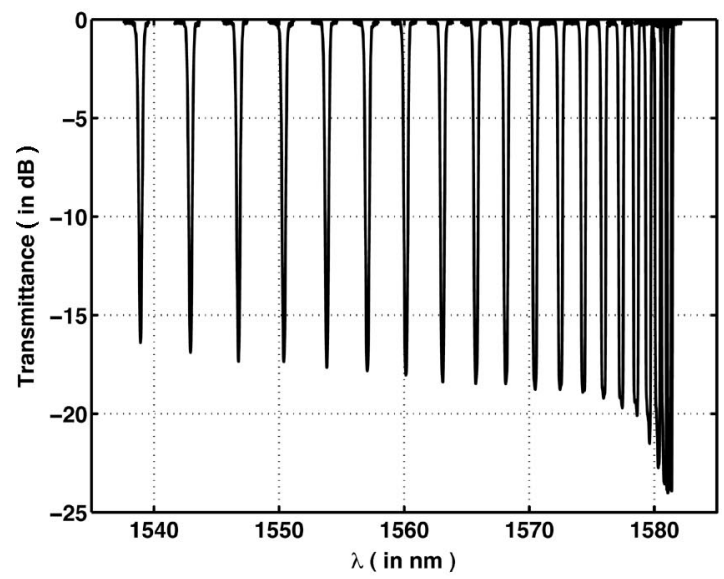

(b)

Fig. 3. Wavelength selectivity curves from normal to oblique incidence in the reflection geometry. The 20 measured curves in (a) and (b) correspond, from right to left, to incident angles $0^{\circ}, 1^{\circ}, 2^{\circ}, \ldots, 19^{\circ}$ outside the glass sample $\left(\approx 0^{\circ}-13.3^{\circ}\right.$ inside the glass $)$. 


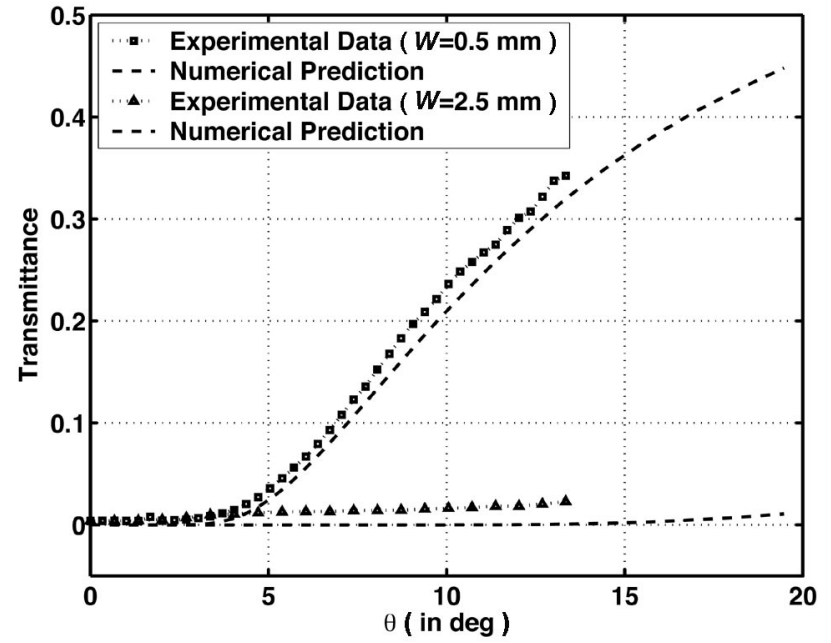

Fig. 4. Summary of the wavelength selectivity measurements and the comparison with numerical simulations. The increasing transmission of the narrow beam at oblique incidence contrasts strongly with the transmission of the expanded beam, which does not increase much at oblique incidence.

diffracted beam and the wavelength of its transmission minimum, defined as the Bragg wavelength $\lambda_{B}$, satisfies the relationship

$$
\lambda_{B}=2 n \Lambda \cos \theta .
$$

The same set of measurements performed for the wide, expanded beam $B_{W}(W=2.5 \mathrm{~mm})$ is summarized in Fig. 3(b). As we can see, the almost constant transmittance for $B_{W}$ at oblique incidence contrasts strongly with the increasing transmittance of $B_{N}$, which is predicted by numerical simulations. The transfer function $H=E_{d}\left(x^{\prime}, y, z^{\prime}=0\right) /$ $E_{i}\left(x^{\prime}, y, z^{\prime}=0\right)$ used for all reflection-geometry simulations is

$$
H=\frac{-j \kappa \sinh s L}{s \cosh s L-j \frac{\Delta \beta}{2} \sinh s L},
$$

where we have set $\mathbf{K}=K, \Delta \beta=2 k \cos \theta-K$, and the coupling constant $\kappa=\pi \Delta n /(\lambda \cos \theta)$. Parameter $s$ is defined as

$$
s=\sqrt{\kappa^{2}-\left(\frac{\Delta \beta}{2}\right)^{2}} .
$$

The quantitative comparison is further summarized in Fig. 4; the numerical simulations (calculated with an index modulation $\Delta n=4.7 \times 10^{-4}$ ) agree well with the experimental data.

\section{Angular Selectivity}

To measure the angular selectivity curves, the incident angle is first set to one of a series of predetermined values. With the wavelength tuned to the appropriate Bragg wavelength $\lambda_{B}$ according to Eq. (6), an angular scan is then performed. Figures 5(a) and 5(b) show the measured angular selectivities for $B_{N}$ and $B_{W}$, respectively. The $0.5 \mathrm{~dB}$ angular bandwidths $\Delta \theta_{\mathrm{BW}}$ are summarized in Fig. 6 along with the numerically simulated results.

Again the minimum transmittance increases much more rapidly for $B_{N}$ than for $B_{W}$ as we tilt the beam from normal to oblique incidence. An interesting feature common to both $B_{N}$ and $B_{W}$ is the dramatic decrease of the angular bandwidth $\Delta \theta_{\mathrm{BW}}$ at oblique incidence.

Some of the angular selectivity curves, as traced out by dashed curves in Figs. 5(a) and 5(b), near normal incidence have a "twin-valley" $(\omega)$ shape. Each of them can be thought of as the "fusion" of two normal, single-dip angular selectivity curves positioned close together. Owing to geometric degeneracy at normal incidence, the effects caused by a positive $\theta$

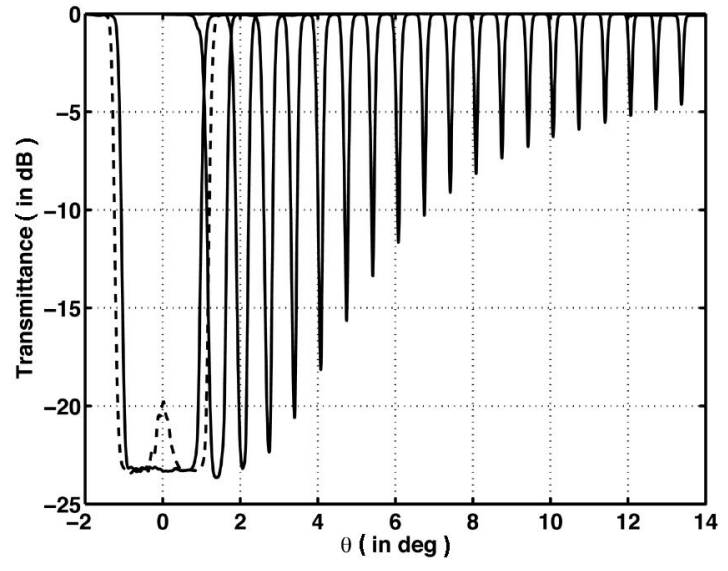

(a)

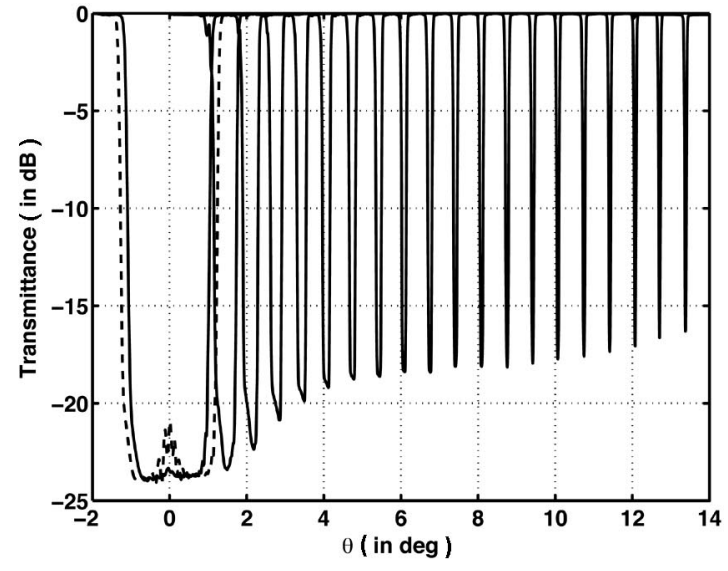

(b)

Fig. 5. Angular selectivity curves from normal to oblique incidence in the reflection geometry. The 20 solid curves in (a) and (b) correspond, from left to right, to incident angles $0^{\circ}, 1^{\circ}, 2^{\circ}, \ldots, 19^{\circ}$ outside the glass sample $\left(\approx 0^{\circ}-13.3^{\circ}\right.$ inside the glass $)$. The dashed curves in both plots are measured for an incident angle of $0.5^{\circ}$ outside the glass. 


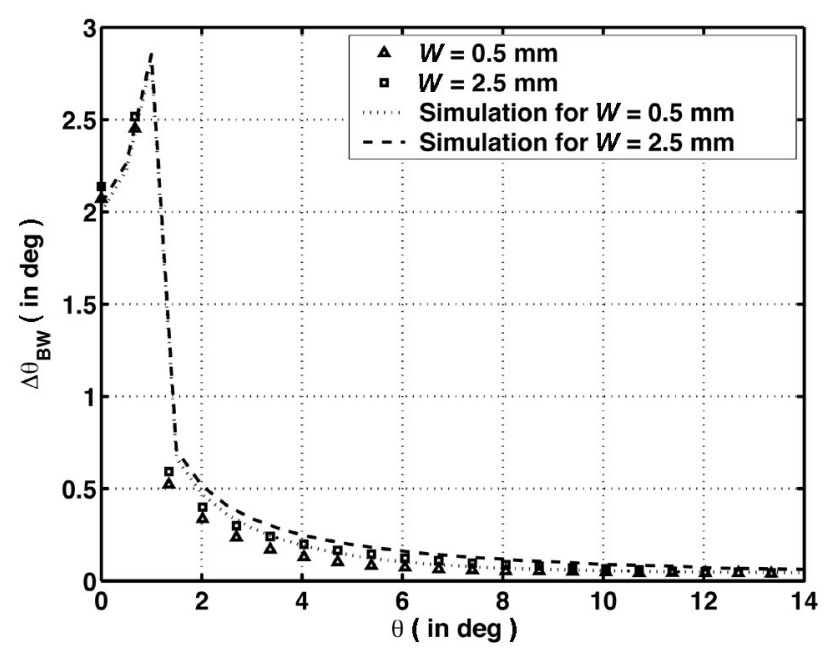

Fig. 6. Summary of the angular selectivity measurements. The $0.5 \mathrm{~dB}$ angular bandwidth, $\Delta \theta_{\mathrm{BW}}$, is plotted against the angle of incidence $\theta$. Numerical simulations are seen to agree well with the experimental data.

are equivalent to those caused by a negative one in the reflection geometry; whenever $\theta$ is small compared with $\Delta \theta_{\mathrm{BW}}$, such "fusion" will inevitably occur. This is the reason why we observe the increase of $\Delta \theta_{\mathrm{BW}}$ prior to its drastic decrease.

The angular and wavelength selectivity curves do not behave independently. The relationships between them can be unraveled if we consider the spatial harmonic components of the incident beam. At normal incidence, the VHG's angular bandwidth $\Delta \theta_{\mathrm{BW}}$ is wide and diffracts most spatial harmonics contained within both $B_{N}$ and $B_{W}$, therefore $\eta$ approaches $100 \%$. If we slightly tilt the incident beam away from the normal, we effectively increase $\Delta \theta_{\mathrm{BW}}$ and almost all spatial harmonics remain strongly diffracted and the wavelength selectivity curve does not change much. Around normal incidence where we get the $\omega$-shaped angular selectivity, the angular and wavelength selectivity of $B_{N}$ differs little from that of $B_{W}$. However, as we tilt the incident beam past an angular thresh- old ( $\approx 1^{\circ}$ in our case), $\Delta \theta_{\mathrm{BW}}$ starts to decrease sharply and a smaller and smaller portion of the spatial harmonic content of $B_{N}$ gets diffracted efficiently by the grating. On the other hand, $B_{W}$ is not affected as much thanks to its narrower spatial frequency spread. This is when $B_{W}$ starts to have a higher diffraction efficiency because part of the energy of $B_{N}$ spills out of the $\Delta \theta_{\mathrm{BW}}$ angular stop band along with the undiffracted spatial components. To put it succinctly, the product of a VHG's angular selectivity curve and the angular spectrum of the incident beam determines the diffraction efficiency and filter shape of the VHG.

\section{Diffracted Beam Profiles}

In Figs. 7(a) and 7(b), we show the experimentally measured and numerically simulated diffracted beam intensity profiles for $B_{N}$ and $B_{W}$, respectively. The legend in Fig. 7(a) applies to both plots. At oblique incidence, we see that the diffracted beam of $B_{N}$ is stretched out from its original Gaussian profile. On the contrary, the diffracted beams of $B_{W}$ maintain their Gaussian-like profiles. This phenomenon is again attributed to the strong angular filtering suffered by $B_{N}$ thanks to the VHG: its wider diffracted beam profiles are the results of less diffracted spatial harmonic components. This effect is not readily observable for $B_{W}$ because most of its spatial harmonics are still diffracted. Again the simulations can accurately predict the measured data.

\section{B. Transmission Geometry}

\section{Experimental Setup}

For the measurements of a VHG in the transmission geometry, we replace $\mathrm{VHG}_{R}$ with $\mathrm{VHG}_{T}$ in Fig. 2 . The only differences are the grating orientation and the position of the detector for the diffraction beam. The intensity profile of the diffracted beams is measured $50 \mathrm{~mm}$ from the output face.

The grating period $\Lambda$ of $\mathrm{VGH}_{T}$ is approximately $5.94 \mu \mathrm{m}$, and the Bragg angle $\theta_{B}$ at $1560 \mathrm{~nm}$ is roughly $5^{\circ}$. The index modulation $\Delta n$ is estimated to

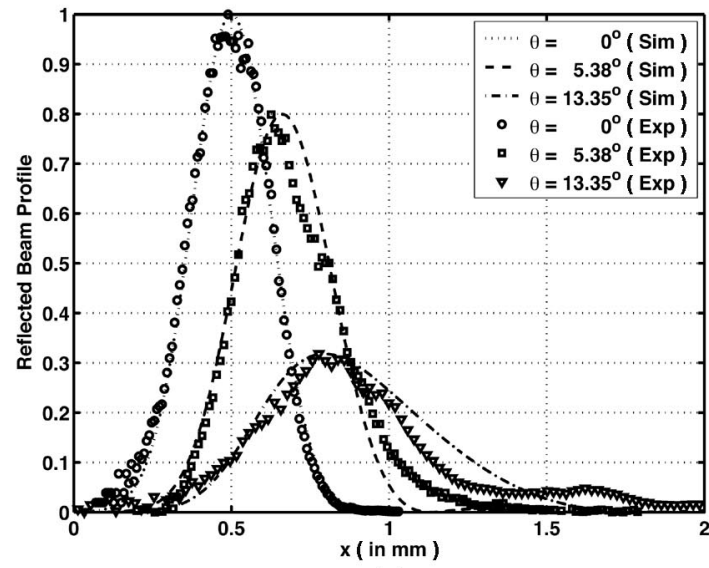

(a)

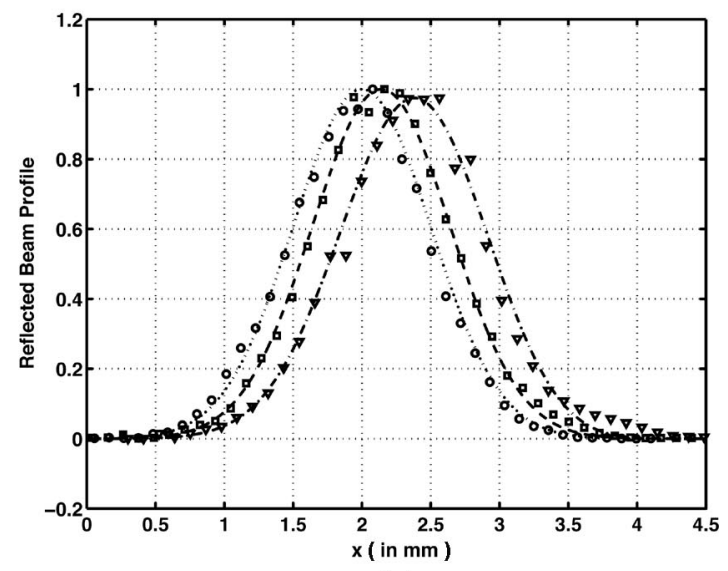

(b)

Fig. 7. Diffracted beam intensity profiles for three incident angles from normal to oblique incidence in the reflection geometry. 


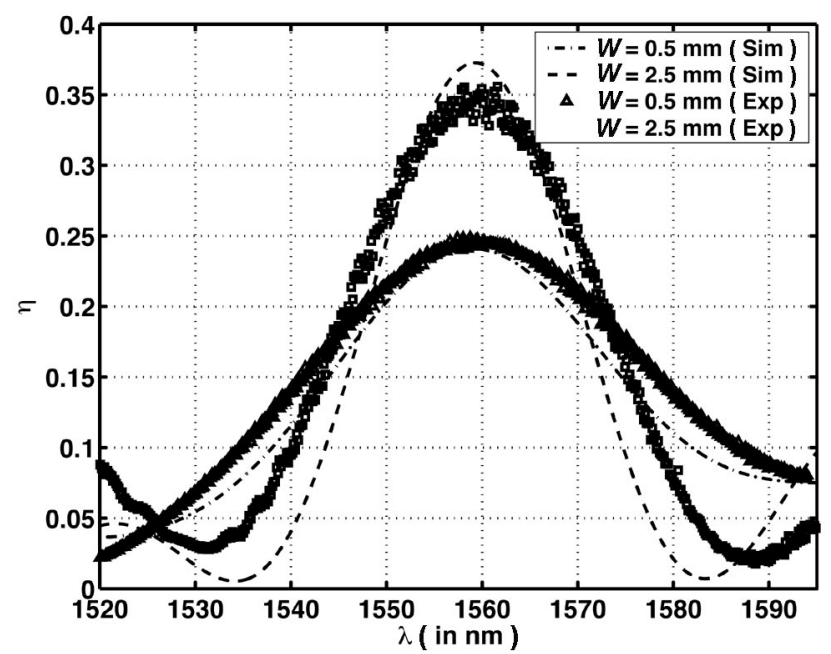

Fig. 8. Wavelength selectivity curves at $\theta \approx 5.1^{\circ}$ in the transmission geometry for beam widths $W=0.5$ and $2.5 \mathrm{~mm}$.

be $4.7 \times 10^{-4}$ and the interaction length $L$ at normal incidence is $3 \mathrm{~mm}$.

\section{Wavelength Selectivity}

The procedure for measuring the wavelength selectivity curves is as follows: we first rotate $\mathrm{VGH}_{T}$ such that $\theta \approx 5.1^{\circ}$ (which is the Bragg angle for $\lambda$ $=1590 \mathrm{~nm}$ ) and then the wavelength is scanned from 1520 to $1600 \mathrm{~nm}$. The resulting selectivity curves for $B_{N}$ and $B_{W}$ are shown together in Fig. 8. Along with the measured data we also plot the numerically simulated curves. The selectivity curves are seen to be quite wide. This is because of the very shallow incident angle (small value of $\theta$ ). The transfer function $H=E_{d}\left(x^{\prime}, y, z^{\prime}=L\right) / E_{i}\left(x^{\prime}, y, z^{\prime}=0\right)$ used for all transmission-geometry simulations is

$$
H=-j \exp \left(j \frac{\Delta \beta}{2} L\right) \frac{\pi \Delta n}{\lambda \cos \theta_{d}} \frac{\sin s L}{s},
$$

where we have $\theta_{d}=\arcsin \left(K / k_{i}-\sin \theta\right)$ and $\Delta \beta=$

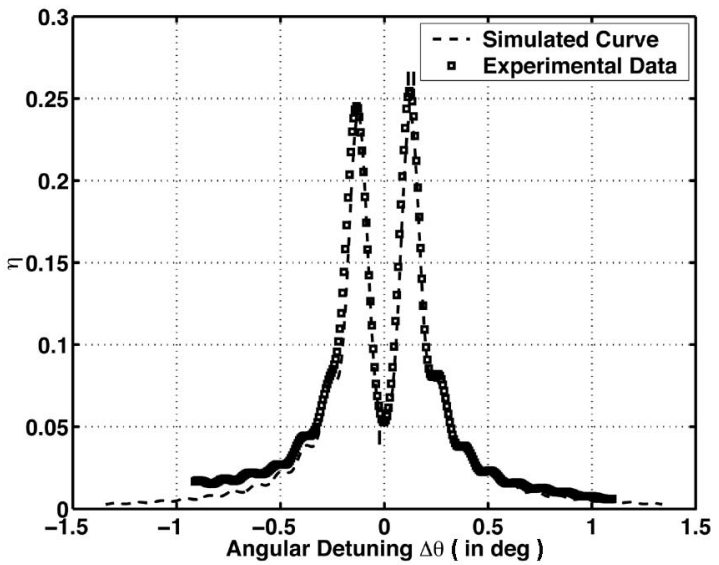

(a) $k\left(\cos \theta-\cos \theta_{d}\right)$, and the parameter $s$ is defined as

$$
s=\sqrt{\kappa^{2}+\left(\frac{\Delta \beta}{2}\right)^{2}} .
$$

For plane waves, $\eta=\sin ^{2}(\pi \Delta n L / \lambda \cos \theta)$ when the Bragg condition is satisfied. We see that $\eta$ reaches $100 \%$ when the parameter $\pi \Delta n L / \lambda \cos \theta$ is equal to $0.5 \pi, 1.5 \pi, \ldots$, . In our experiments, this parameter is $\approx 0.91 \pi$ and therefore the maximum value of $\eta$ occurs when a phase mismatch is present instead of when Bragg matched. This behavior transpires because part of the diffracted beam is coupled back into the transmitted beam and $\eta$ is reduced.

\section{Angular Selectivity}

The angular selectivity curves are measured by scanning around the Bragg angle when the wavelength is fixed at $1560 \mathrm{~nm}$. The measured curves for $B_{N}$ and $B_{W}$ along with the theoretically predicted responses are plotted in Figs. 9(a) and 9(b).

We do not get the maximum diffracted intensity at the Bragg angle but at a certain amount of angular detuning $\left(\Delta \theta=\theta-\theta_{B} \approx \pm 0.13^{\circ}\right)$. As we increase the absolute angular detuning $|\Delta \theta|$ from $0^{\circ}$, our angular selectivity curves are traced out almost symmetrically around the Bragg angle $\theta_{B}$. The numerical simulation matches the experimental data so well that even the absence and/or presence of a local "bump" at $\theta_{B}$ for $B_{N} / B_{W}$ is accurately depicted.

\section{Diffracted Beam Profiles}

We plot two measured diffracted beam profiles for both $B_{N}$ and $B_{W}$ in Fig. 10. The normalized intensity profiles (a), (b), (c), and (d) correspond to indices in Roman numerals from I to IV as marked in Figs. 9(a) and $9(\mathrm{~b})$. While the diffracted beam profiles (b), (c), and (d) remain Gaussian (as predicted by the simulations), the beam profile (a) shows a dip in the center. The explanation for this interesting phenomenon is that the phase relationship between the constituent diffracted plane-wave components dictates de-

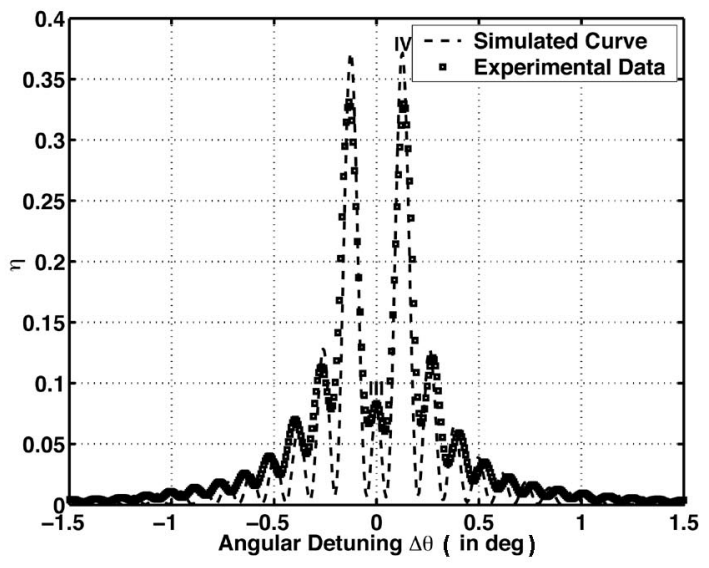

(b)

Fig. 9. Angular selectivity curves in the transmission geometry around Bragg angle $\theta_{B} \approx 5^{\circ}$. 

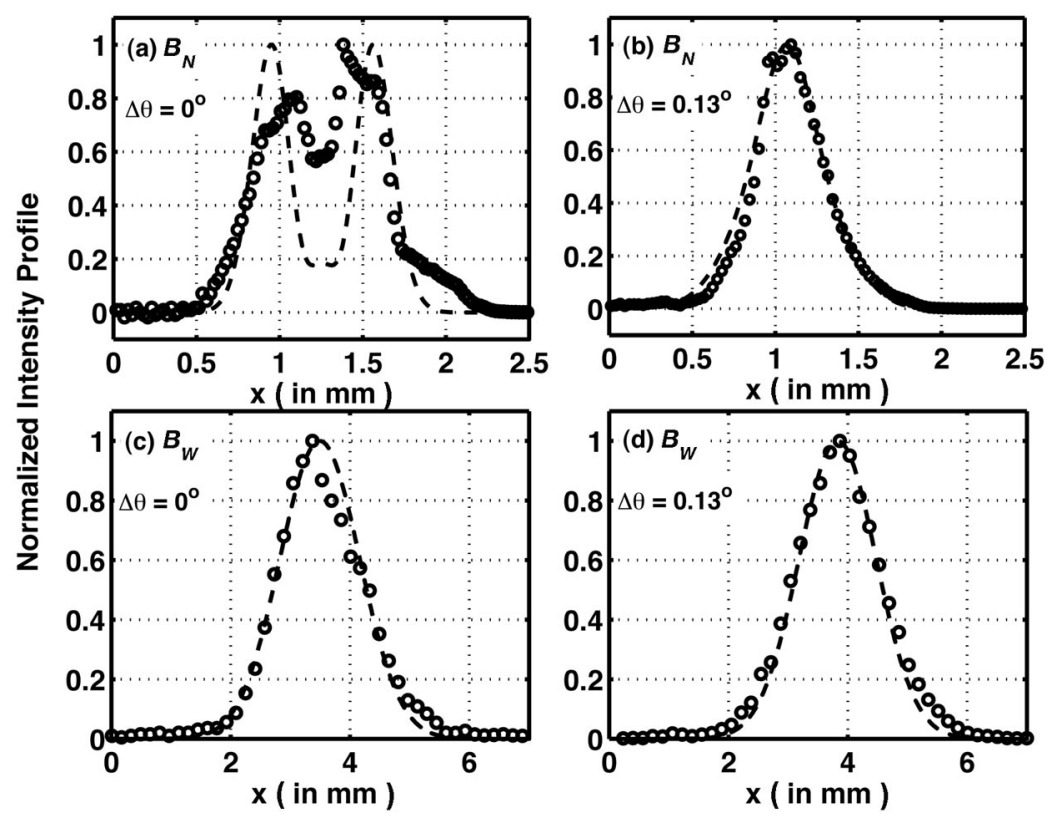

Fig. 10. Normalized diffracted intensity beam profiles in the transmission geometry around Bragg angle $\theta_{B} \approx 5^{\circ} ; \Delta \theta=\theta-\theta_{B}$. All beam profiles are measured $50 \mathrm{~mm}$ from the output face. The circles represent experimental measurements and the dashed lines are the numerical simulations. structive interference at the center of the diffracted beam.

\section{Conclusion}

The angular selectivity of a volume holographic grating determines its beam-width-dependent behaviors. Whenever the constituent plane-wave components of the incident beam are selectively diffracted by the volume grating, deviation from the ideal response predicted by the plane-wave solution will take place, especially for narrow optical beams.

Taking advantage of the technique of Fourier decomposition combined with plane-wave solutions to the VHGs proves to be extremely useful for accurately characterizing and predicting the diffraction effects experienced by a finite incident beam owing to a VHG. Such characterization will be useful for evaluating the performance of optical systems employing VHGs, determining the optimal parameters at the design phase, and improving existing optical systems.

This work was supported by the NSF Engineering Research Centers Program for Neuromorphic Systems Engineering under award EEC-9402726.

\section{References}

1. L. B. Glebov, "Kinetics modeling in photosensitive glass," Opt. Mater. 25, 413-418 (2004).

2. G. C. Valley, M. B. Klein, R. A. Mullen, D. Rytz, and B. Wechsler, "Photorefractive materials," Annu. Rev. Mater. Sci. 18, 165-188 (1988).

3. J. E. Ludman, J. R. Riccobono, N. O. Reinhard, I. V. Semenova, Y. L. Korzinin, and S. M. Shahriar, "Very thick holographic nonspatial filtering of laser beams," Opt. Eng. 36, 1700-1705 (1997).

4. H. Kogelnik, "Coupled wave theory for thick hologram gratings," Bell Syst. Tech. J. 48, 2909-2947 (1969).

5. J. W. Goodman, Fourier Optics (McGraw-Hill, 1996).

6. P. Yeh, Optical Waves in Layered Media (Wiley, 1991).

7. D. Psaltis, D. Brady, X. G. Xu, and S. Lin, "Holography in artificial neural networks," Nature 343, 325-330 (1990).

8. M. Levene, G. J. Steckman, and D. Psaltis, "Method for controlling the shift invariance of optical correlators," Appl. Opt. 38, 394-398 (1999).

9. D. Gabor, "Associative holographic memories," IBM J. Res. Dev. 13, 156-159 (1969).

10. D. Psaltis and F. Mok, "Holographic memories," Sci. Am. 273, 70-76 (1995).

11. G. A. Rakuljic and V. Leyva, "Volume holographic narrowband optical filter," Opt. Lett. 18, 459-461 (1993).

12. S. Breer and K. Buse, "Wavelength demultiplexing with volume phase holograms in photorefractive lithium niobate," Appl. Phys. B 66, 339-345 (1998).

13. V. Polo, B. Vidal, J. L. Corral, and J. Marti, "Novel tunable photonic microwave filter based on laser arrays and $\mathrm{N} \times \mathrm{N}$ AWG-based delay lines," IEEE Photon. Technol. Lett. 15, 584586 (2003)

14. S. Y. Li, N. Q. Ngo, S. C. Tjin, P. Shum, and J. Zhang, "Thermally tunable narrow-bandpass filter based on a linearly chirped fiber Bragg grating," Opt. Lett. 29, 29-31 (2004).

15. G. A. Ball and W. W. Morey, "Compression-tuned singlefrequency Bragg grating fiber laser,” Opt. Lett. 19, 1979-1981 (1994).

16. H. T. Hsieh, G. Panotopoulos, M. Liger, Y. C. Tai, and D. Psaltis, "Athermal holographic filters," IEEE Photon. Technol. Lett. 16, 177-179 (2004). 